\title{
No increase in Kidney Injury Molecule-1 and Neutrophil Gelatinase-Associated Lipocalin excretion following intravenous contrast enhanced-CT
}

\author{
Judith Kooiman • Wilke R. van de Peppel • Yvo W. J. Sijpkens • Harald F. H. Brulez • \\ P. M. de Vries - Mioara A. Nicolaie • H. Putter • Menno V. Huisman • \\ W. van der Kooij • Cees van Kooten • Ton J. Rabelink
}

Received: 18 August 2014 / Revised: 31 October 2014 / Accepted: 21 January 2015 / Published online: 15 March 2015

(C) The Author(s) 2015. This article is published with open access at Springerlink.com

\begin{abstract}
Objectives To analyze kidney injury molecule-1 (KIM-1) and neutrophil gelatinase-associated lipocalin (N-GAL) excretion post-intravenous contrast enhanced-CT (CE-CT) in patients with chronic kidney disease (CKD).

Methods Patients were enrolled in a trial on hydration regimes to prevent contrast-induced acute kidney injury (CI-AKI). Blood and urine samples were taken at baseline, $4-6$, and 48 - 96 h post CE-CT. Urinary KIM-1 and N-GAL values were normalized for urinary creatinine levels, presented as medians with $2.5-97.5$ percentiles.

Results Of the enrolled 511 patients, 10 (2\%) were lost to follow-up. CI-AKI occurred in $3.9 \%$ of patients $(20 / 501)$.
\end{abstract}

J. Kooiman $(\varangle) \cdot$ W. R. van de Peppel $\cdot$ M. V. Huisman

Department of Thrombosis and Haemostasis, Leiden University

Medical Center, Albinusdreef 2, Postzone C7-Q, 2333

ZA Leiden, The Netherlands

e-mail: j.kooiman@lumc.nl

Y. W. J. Sijpkens

Department of Nephrology, Bronovo Hospital, The Hague, The Netherlands

H. F. H. Brulez

Department of Nephrology, Sint Lucas Andreas Hospital,

Amsterdam, The Netherlands

P. M. de Vries

Department of Vascular Surgery, St. Antonius Hospital,

Nieuwegein, The Netherlands

M. A. Nicolaie $\cdot$ H. Putter

Department of Medical Statistics and Bioinformatics, Leiden

University Medical Center, Leiden, The Netherlands

J. Kooiman · W. van der Kooij • C. van Kooten • T. J. Rabelink Department of Nephrology, Leiden University Medical Center, Leiden, The Netherlands
Median KIM-1 values were $1.2(0.1-7.7)$ at baseline, 1.3 $(0.1-8.6)$ at $4-6 \mathrm{~h}$, and $1.3 \mathrm{ng} / \mathrm{mg}(0.1-8.1)$ at $48-96 \mathrm{~h}$ post CE-CT $(P=0.39)$. Median N-GAL values were 41.0 (4.4 $-3,174.4), 48.9(5.7-3,406.1)$, and $37.8 \mu \mathrm{g} / \mathrm{mg}(3.5-3$, $200.4)$, respectively $(P=0.07)$. The amount of KIM-1 and NGAL excretion in follow-up was similar for patients with and without CI-AKI ( $P$-value KIM-1 $0.08, P$-value N-GAL 0.73 ). Neither patient characteristics at baseline including severe $\mathrm{CKD}$, medication use, nor contrast dose were associated with increased excretion of KIM-1 or N-GAL during follow-up. Conclusion KIM-1 and N-GAL excretion were unaffected by CE-CT both in patients with and without CI-AKI, suggesting that CI-AKI was not accompanied by tubular injury.

Key Points

- KIM-1 and N-GAL excretion were unaffected by intravenous contrast-enhanced CT (CE-CT).

- Patient or procedure characteristics were not associated with increased KIM-1 or N-GAL excretion.

- Performance of CE-CT in CKD patients is likely to be safe.

Keywords Acute kidney injury $\cdot$ Contrast media $\cdot$ Renal insufficiency, chronic $\cdot$ Biological markers $\cdot$ Multidetector computed tomography

\section{Introduction}

Contrast-induced acute kidney injury (CI-AKI) is an acute decline in renal function following administration of iodinated contrast media $[1,2]$. CI-AKI occurs in $5-6 \%$ of patients undergoing intravenous contrast enhanced computed tomography (CE-CT), a very common procedure worldwide [3, 4]. Although the definition of CI-AKI is the subject of debate, all proposed criteria are based on changes in serum creatinine within a few days following contrast administration [5]. However, 
serum creatinine is regarded as a non-specific marker for CI-AKI since several mechanisms (i.e. use of medication, hemodynamics, and comorbidity such as peripheral artery disease or diabetes) can also influence its value $[1,6,7]$. The clinical significance of serum creatinine changes post CE-CT has become disputable after publication of studies suggesting that fluctuations in serum creatinine occur as frequent in patients undergoing CE-CT, as in those not receiving contrast media [8, 9]. However, physicians are still concerned about the risk of CI-AKI and are hesitant to use CE-CT in their diagnostic workup, especially in patients with pre-existing chronic kidney disease (CKD) [8]. Knowledge about the risk of renal injury post-CE-CT is, therefore, of clinical importance and may be derived from studies measuring biomarkers of acute kidney injury in the context of CI-AKI, such as kidney injury molecule-1 (KIM-1) and neutrophil gelatinaseassociated lipocalin (N-GAL). These biomarkers have been proven to be predictive of CI-AKI in the setting of percutaneous coronary interventions requiring intra-arterial contrast administrations [10-16]. Yet, although N-GAL excretion has been analyzed in a small cohort of patients undergoing CE-CT, these biomarkers have not been studied thoroughly within this population. Hence, the aim of our study was to investigate KIM-1 and N-GAL excretion and their association with the occurrence CI$\mathrm{AKI}$ after CE-CT in patients with pre-existing CKD.

\section{Methods}

Study patients were enrolled in a randomized controlled trial on CI-AKI preventing hydration regimes between January 2010 and June 2012 in four Dutch hospitals (Leiden University Medical Center, Leiden, Bronovo Hospital, The Hague, St. Lucas Andreas Hospital, Amsterdam, and St. Antonius Hospital, Nieuwegein). Patients electively underwent CE-CT and were randomized to either $250 \mathrm{ml}$ $1.4 \%$ sodium bicarbonate hydration $1 \mathrm{~h}$ prior to CE-CT or standard treatment with $1,000 \mathrm{ml} 0.9 \%$ saline during 4 to $12 \mathrm{~h}$ prior to and after CE-CT. No other CI-AKI preventive treatments were used, such as administration of $\mathrm{N}$-acetylcysteine. Patients were 18 years or older, eligible to receive the fluid challenge of saline hydration, and had an estimated glomerular filtration rate $(\mathrm{eGFR})<60 \mathrm{ml} / \mathrm{min}$. eGFR values were calculated using the abbreviate modification of diet in renal disease (MDRD) formula [17]. Exclusion criteria were documented allergy for iodinated contrast media, pregnancy, previous contrast administrations within 7 days prior to CE-CT, and hemodynamic instability. In addition to the general exclusion criteria for the trial, patients in whom CT was cancelled or unexpectedly performed without intravenous contrast administration were also excluded for the current analysis. Type of contrast media used at time of CE-CT was according to clinical practice in the participating hospitals and varied between Iomeprol (Iomeron, Bracco Imaging, Milan, Italy), Iobitridol
(Xenetix, Guerbet, Aulnay-sous-Bois, France), or Iodixanol (Visipaque, GE Healthcare, Chalfort St. Giles, UK). An independent data and safety monitoring board periodically reviewed study outcomes. All patients provided written informed consent and the protocol of the trial was approved by the institutional review boards of each of the participating centres. The trial was performed according to the declaration of Helsinki and was registered with the Netherlands Trial Register, NTR 2149.

\section{Materials}

Serum and urine samples were collected prior to hydration, and once between $4-6$ and $48-96 \mathrm{~h}$ post-CE-CT for analyses of serum creatinine and urinary creatinine, KIM-1 and NGAL levels. Urinary creatinine levels were used to correct KIM-1 and N-GAL values for dilution as a result of the two hydration regimes [18]. Therefore, analyses were made using $\mathrm{N}$-GAL/urine creatinine and KIM-1/urine creatinine ratios, which will be revered to as KIM-1 (in ng/mg) and N-GAL $(\mu \mathrm{g} / \mathrm{mg})$ excretion. Serum and urine samples were also collected 2 months post-CE-CT for patients developing CI-AKI, to determine whether renal function had recovered. Furthermore, information on patient characteristics at baseline, use of nephrotoxic medication and injected contrast volumes were collected.

\section{Outcomes and Definitions}

Primary outcomes of our study were relative changes in KIM1 and N-GAL excretion post CE-CT as compared with baseline. Furthermore, we studied whether changes in KIM-1 and N-GAL excretion post-CE-CT correlated to changes in serum creatinine or the occurrence of CI-AKI. CI-AKI was defined by an increase in serum creatinine over $25 \%$ or $44 \mu \mathrm{mol} / \mathrm{L}$ $(0.5 \mathrm{mg} / \mathrm{L})$ within $48-96 \mathrm{~h}$ post-CE-CT. Renal function was considered recovered if the increase in serum creatinine 2 months post-CE-CT no longer exceeded these thresholds.

\section{Laboratory Methods}

All samples were stored at $-80^{\circ} \mathrm{C}$. Serum and urine creatinine levels were analyzed using Roche Diagnostics, Mannheim, Germany. Urinary KIM-1 and N-GAL values were analyzed using ELISA (R\&D systems, DuoSets Human TIM-1/KIM-1/ HAVCR and Lipocalin-2/N-GAL, MN, USA).

\section{Statistical Analyses}

KIM-1 and N-GAL excretion at $4-6$ and $48-96 \mathrm{~h}$ post-CECT were compared with baseline using linear mixed models based on log-transformed KIM-1 and N-GAL values as they 
were non-normally distributed among the population. The base linear mixed model contained random subject effects and fixed effects of time (categorical), randomization, and their interaction. To study whether baseline conditions or contrast dose were associated with changes in KIM-1 and N-GAL excretion during follow-up, these factors and their interaction with time were added to the base model. Correlation studies were performed on the association between changes in serum creatinine and in KIM- 1 and N-GAL excretion at $4-6$ and 48 $-96 \mathrm{~h}$ post-CE-CT compared with baseline, using Spearman's correlation tests, and reported as correlation terms with corresponding $P$-values. The area under the receiver operating curve (ROC) was calculated under nonparametric assumption, to analyze the predictive value of KIM-1 and N-GAL excretion for the occurrence of CI-AKI measured at baseline, $4-6$ and $48-96$ h post-CE-CT. $P$-values $<0.05$ were considered statistically significant. All statistical analyses were performed using SPSS version 20.

\section{Results}

Study population

A total of 570 patients provided written informed consent. Of those, 22 patients withdrew their consent and CT was performed without intravenous contrast administration or cancelled in 37 patients. As a result, the total population for this analysis comprises 511 patients of whom $10(2.0 \%)$ where lost to follow-up for the endpoint of CI-AKI. Patient characteristics at baseline are reported in Table 1. Mean relative increase in serum creatinine $48-96 \mathrm{~h}$ post-CE-CT compared with baseline was $1.2 \%$ (SD $13.3 \%$ ). CI-AKI occurred in $3.9 \%$ of patients $(20 / 501,95 \%$ CI $2.6-6.1 \%)$. Renal function recovered within 2 months post-CE-CT in 13 out of 19 (68.0\%) patients diagnosed with CI-AKI and one patient was lost to follow-up. None of the patients developing CI-AKI had a need for renal replacement therapy within 2 months after CE-CT.

\section{N-GAL and KIM-1 excretion}

Baseline KIM-1 and N-GAL values correlated well with KIM-1 (Spearman's rho 0.83, $P<0.001$ ) and N-GAL excretion adjusted for dilution by urine creatinine (Spearman's rho 0.87, $P<0.001$ ) (Fig. 1 a, b). Median KIM-1 and N-GAL excretion at baseline, $4-6 \mathrm{~h}$ and $48-96 \mathrm{~h}$ post-CE-CT are reported in Table 2. Figures 2 and 3 reflect KIM- 1 and N-GAL excretion in patients with and patients without CI-AKI at A) baseline, B) $4-6 \mathrm{~h}$, and C) $48-96 \mathrm{~h}$ post-intravenous contrast-enhanced computed tomography. Both KIM-1 and NGAL excretion remained unchanged during follow-up within the total population. Additionally, the relative change in KIM-
Table 1 Patient characteristics at baseline

\begin{tabular}{|c|c|c|}
\hline $\mathrm{N}$ & 511 & \\
\hline Mean age, years & 72.3 & $(9.4)$ \\
\hline Sex, male & 308 & $(60.3)$ \\
\hline Outpatients & 478 & $(93.5)$ \\
\hline Mean eGFR $\mathrm{ml} / \mathrm{min}$ & 50.7 & $(13.8)$ \\
\hline $\mathrm{eGFR}>45 \mathrm{ml} / \mathrm{min}$ & 354 & $(69.3)$ \\
\hline eGFR $30-45 \mathrm{ml} / \mathrm{min}$ & 121 & $(23.7)$ \\
\hline $\mathrm{eGFR}<30 \mathrm{ml} / \mathrm{min}$ & 36 & $(7.0)$ \\
\hline Hypertension & 256 & $(50.1)$ \\
\hline Diabetes mellitus & 137 & $(26.8)$ \\
\hline Peripheral artery disease & 168 & $(32.9)$ \\
\hline Coronary artery disease & 175 & $(34.2)$ \\
\hline Congestive heart failure & 87 & $(17.0)$ \\
\hline Primary renal or urological disease & 224 & $(43.8)$ \\
\hline \multicolumn{3}{|l|}{ Medication } \\
\hline NSAIDs & 26 & $(5.1)$ \\
\hline Diuretics & 234 & $(45.8)$ \\
\hline ACE-inhibitors & 205 & $(40.1)$ \\
\hline Angiotensin II receptor blockers & 101 & $(19.8)$ \\
\hline Chemotherapy & 11 & $(2.2)$ \\
\hline Preprocedural stop of medication & 117 & $(22.9)$ \\
\hline \multicolumn{3}{|l|}{ Type of CT-scan } \\
\hline CT abdomen & 167 & $(32.7)$ \\
\hline CT thorax & 46 & $(9.0)$ \\
\hline CT angiography & 190 & $(37.2)$ \\
\hline CT thorax-abdomen & 29 & $(5.7)$ \\
\hline CT kidney-pelvis & 43 & $(8.4)$ \\
\hline Other & 36 & $(7.05)$ \\
\hline Mean contrast volume, $\mathrm{ml}$ & 105.2 & $(21.3)$ \\
\hline
\end{tabular}

Data are presented as mean (SD), or $\mathrm{n}(\%)$

$e G F R$, estimated glomerular filtration rate; $C K D$, chronic kidney disease; NSAIDs, non-steroidal anti-inflammatory drugs

1 and N-GAL excretion during follow-up was similar for patients with and without CI-AKI ( $P$-value for KIM-1 excretion $=0.08$ and for N-GAL excretion $=0.73$ ), suggesting that CI-AKI post-CE-CT was not associated with tubular epithelial damage. No correlations were found between an increase in serum creatinine and N-GAL excretion $4-6 \mathrm{~h}$ (correlation term $-0.12, P=0.18$ ), and $48-96 \mathrm{~h}$ post-CE-CT (correlation term -0.04, $P=0.51$ ), or between an increase in serum creatinine and KIM-1 excretion at the same time points (correlation term $0.07, P=0.17$, and $0.08, P=0.12$, respectively). Regression analysis was performed to identify subgroups of patient susceptible for changes in KIM-1 and N-GAL excretion post-CE-CT, the results of which are reported in Table 3. Neither patient characteristics at baseline including severe CKD (i.e. eGFR $<30 \mathrm{ml} / \mathrm{min}$ ), medication use, type of hydration regime, nor contrast dose were associated with changes in KIM-1 or N-GAL excretion during follow-up. 
a

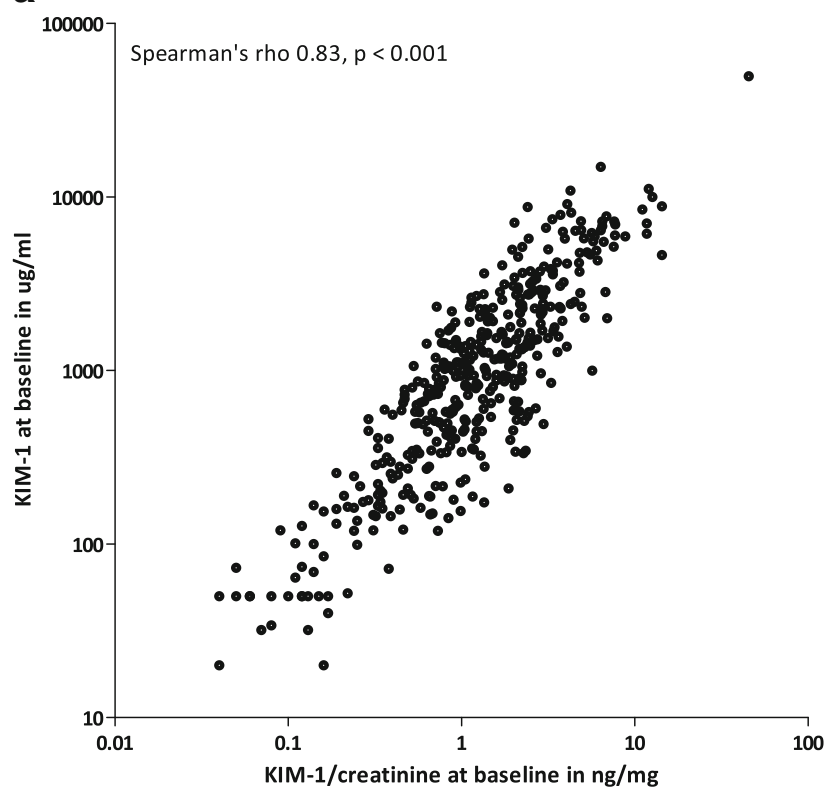

b

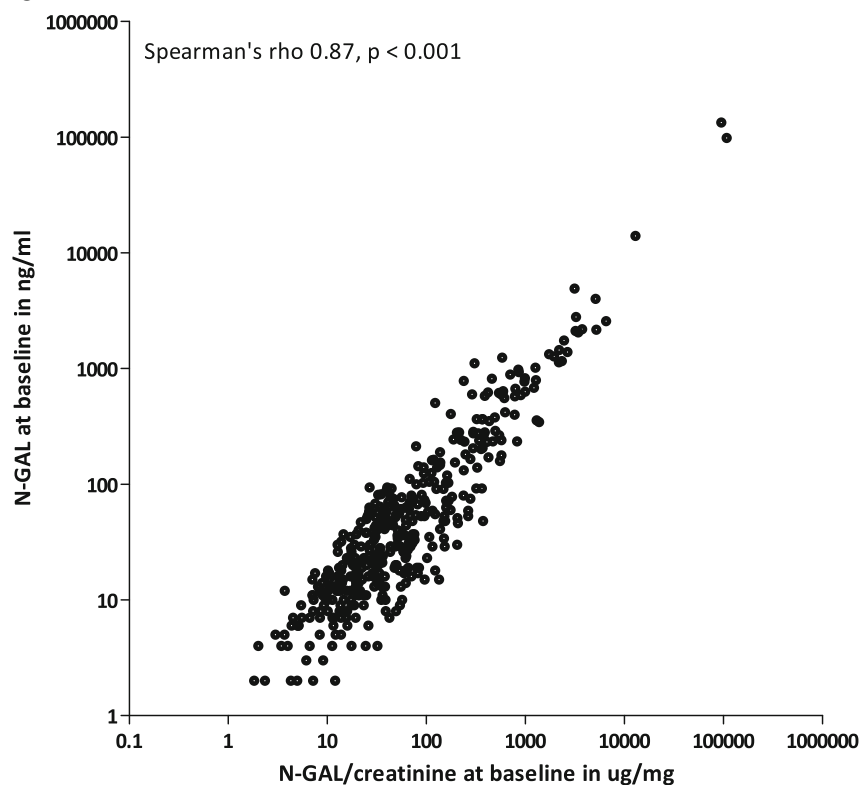

Fig. 1 Correlation between $A$ ) KIM-1 and KIM-1/urine creatinine at baseline, and $B$ ) N-GAL and N-GAL/urine creatinine at baseline

Diagnostic test characteristics

We calculated the diagnostic value of KIM-1 and N-GAL excretion at baseline, $4-6$ and $48-96 \mathrm{~h}$ post-CE-CT for the endpoint of CI-AKI (Table 4). Overall, the performance of the biomarkers was poor, with the highest area under the ROC for N-GAL excretion measured between $48-96 \mathrm{~h}$ postCE-CT (area $0.63,95 \%$ CI $0.45-0.80$ ).

\section{Discussion}

In the present study, we show that KIM-1 and N-GAL excretion were unaffected by CE-CT in CKD patients both with and without CI-AKI. This finding suggests that CE-CT was not accompanied by tubular injury.

The American College of Radiology and the European Society of Urology and Radiology guidelines on CI-AKI prevention state that the risk of CI-AKI is lower after CE-CT compared with intra-arterial contrast administration and that therefore, preventive hydration can be withheld in either all CKD patients [19], or at least in those with CKD stage 3A or less (i.e. eGFR $>45-60 \mathrm{ml} / \mathrm{min}$ ) [2] undergoing CE-CT. The level of evidence for these guideline recommendations is low, as they were based on retrospective studies comparing patients undergoing CE-CT with a control group not receiving iodinated contrast media, showing similar risks of acute kidney injury in both groups [9, 20-23]. However, these studies were prone for confounding by factors associated with the indication for contrast media use despite their attempts to adjust for this matter, and their results are, therefore, difficult to generalize into clinical practice [24].

The current understanding of the pathophysiology of CIAKI is based on tubular injury due to direct toxic effects of contrast agents and secondary tubular injury due to ischemia. We used a valid method to study contrast media induced tubular injury, by measuring KIM-1 and N-GAL excretion post-CE$\mathrm{CT}$, two well-known biomarkers of CI-AKI as shown by several studies among patients undergoing percutaneous coronary interventions [11, 13-16]. Our study results suggest that in both CKD patients with and without CI-AKI, signals for tubular injury post-CE-CT are lacking. Hence, changes in serum creatinine post-CE-CT might be harmless, and possibly the result of interplay between contrast media-induced vasoconstriction [1],

Table 2 KIM-1 and N-GAL excretions during follow-up

\begin{tabular}{lclccccc}
\hline & Baseline & & \multicolumn{2}{c}{$4-6$ h post-CE-CT } & 48-96 h post-CE-CT & $P$-value \\
\hline KIM-1 ratio in ng/mg* & 1.2 & $(0.1-7.7)$ & 1.3 & $(0.1-8.6)$ & 1.3 & $(0.1-8.1)$ \\
N-GAL in ug/mg $\dagger$ & 41.0 & $(4.4-3,174.4)$ & 48.9 & $(5.7-3,406.1)$ & 37.8 & $(3.5-3,200.4)$ & 0.39 \\
\hline
\end{tabular}

Data are presented as median with $2.5-97.5$ percentiles

*Values available at baseline in $431 / 511$, at $4-6 \mathrm{~h}$ in $440 / 511$, and at $48-96 \mathrm{~h}$ post-CE-CET in 401/511

$\dagger$ Values available at baseline in $417 / 511$, at $4-6 \mathrm{~h}$ in $419 / 511$, and at $48-96 \mathrm{~h}$ post-CE-CT in $388 / 511$ 

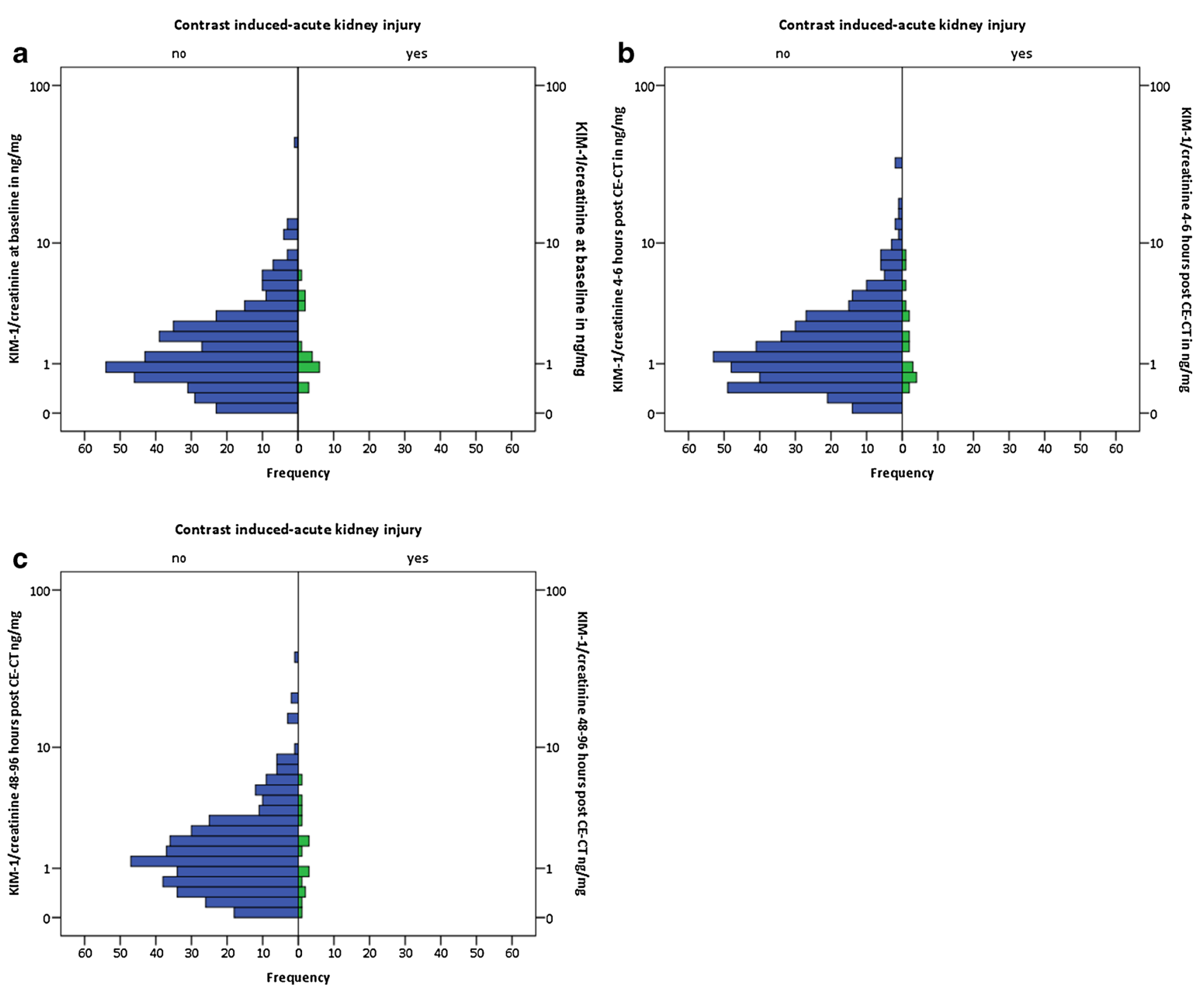

Fig. 2 Figures reflect KIM-1 excretions in patients with (in green) and patients without (in blue) contrast-induced acute kidney injury at $A$ ) baseline, $B$ ) 4 $-6 \mathrm{~h}$, and C) $48-96 \mathrm{~h}$ post-intravenous contrast-enhanced computed tomography

daily intra-individual variations in serum creatinine [24], and co-morbidity affecting serum creatinine values. Our study, therefore, challenges the creatinine-based definition of CI-AKI.

Only two prior studies reported on N-GAL expression post CE-CT and found significant increases in urinary N-GAL after 6 and 8 h, respectively $[25,26]$. However, in the first study, CI-AKI occurred in 23/60 (23\%) patients, which is much higher than the $5-6 \%$ reported by two meta-analyses and the $3.9 \%$ observed in our study $[4,27]$. It is, therefore, questionable whether this study is representative of the general CKD population undergoing CE-CT in daily practice. The second study only included inpatients $(n=47)$, who might be more prone to develop AKI due to other causes than contrast media administration, which was also reflected by the $8.5 \%$ incidence of CI-AKI [26].

The KIM-1 receptor has been proposed to have two different functions [28]. First, KIM-1 serves as a phagocytic receptor on tubular cells contributing to repair processes [28, 29]. Second, KIM-1 might also have an inflammatory modulating role attracting macrophages, monocytes, and fibroblast to the region where epithelium cells are damaged [28]. The exact working mechanism of N-GAL is unclear, although it has been proposed that N-GAL limits tubular injury by modulation of cellular responses such proliferation, apoptosis, and differentiation [30, 31].

In contrast with our results, studies on KIM-1 and N-GAL excretion after intra-arterial contrast injections (not intravenous such as for CE-CT) did find increased levels of these biomarkers during follow-up [11, 13-16]. One of these studies reported absence of an association between N-GAL excretion and adverse clinical outcomes after coronary angiography, whereas the other studies did not report results on the association between increased KIM-1 or N-GAL excretion and adverse clinical outcomes. Therefore, the clinical 

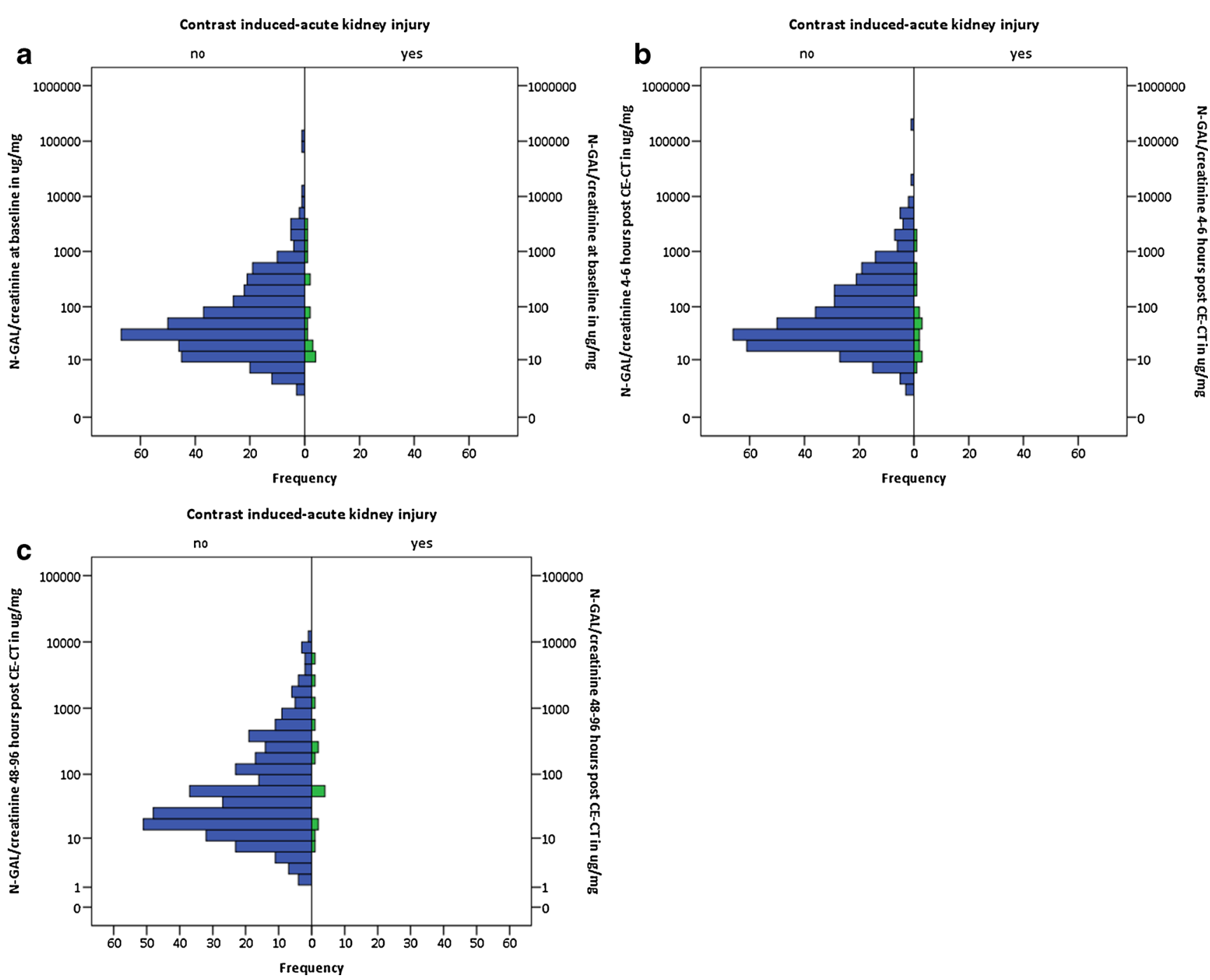

Fig. 3 Figures reflect N-GAL excretions in patients with (in green) and patients without (in blue) contrast-induced acute kidney injury at $A$ ) baseline, $B$ ) $4-6 \mathrm{~h}$, and C) $48-96 \mathrm{~h}$ post-intravenous contrast-enhanced computed tomography

relevance of the increased KIM-1 or N-GAL excretion found after intra-arterial contrast administration in terms of longterm changes in renal function remains unclear.

Our study results show no increase in KIM-1 and N-GAL excretion post-CE-CT and absence of a predictive value of these biomarkers for the development of CI-AKI, which is in contradiction to the literature on N-GAL and KIM-1 excretion after intra-arterial contrast injections. This can be explained as follows. First, percutaneous coronary intervention and other procedures requiring intra-arterial contrast administrations bring along procedural related risk factors for AKI, such as cholesterol emboli shedding into the renal vasculature by catheterization of the descending aorta [32-34]. Second, patients undergoing percutaneous coronary interventions might experience hemodynamic instability as a pre-renal cause of AKI, since acute myocardial infarction is one of the main indications for this procedure. Third, suprarenally injected contrast boluses, as used for radial percutaneous coronary interventions, might be more concentrated and, therefore, nephrotoxic than intravenously injected boluses when they arrive at the level of the kidney. Fourth, patients undergoing CE-CT might differ from patients undergoing intra-arterial contrast administrations in terms of the presence of comorbidity at risk of developing CI-AKI, such as diabetes mellitus [35]. These differences in patient characteristics may also affect KIM-1 and N-GAL expression after administration of iodinated contrast media. Although the results of our study can't designate by which mechanism the risk of tubular injury varies after intravenous and intra-arterial contrast administration, they do suggest that the performance of CE-CT in CKD patients is likely to be safe. This finding endorses the recommendation of the CI-AKI guideline of the American College of Radiology to withhold preventive hydration in CKD patients undergoing CE-CT.

Our study, therefore, adds clinically relevant information to the research field. To our knowledge, this is the first study 


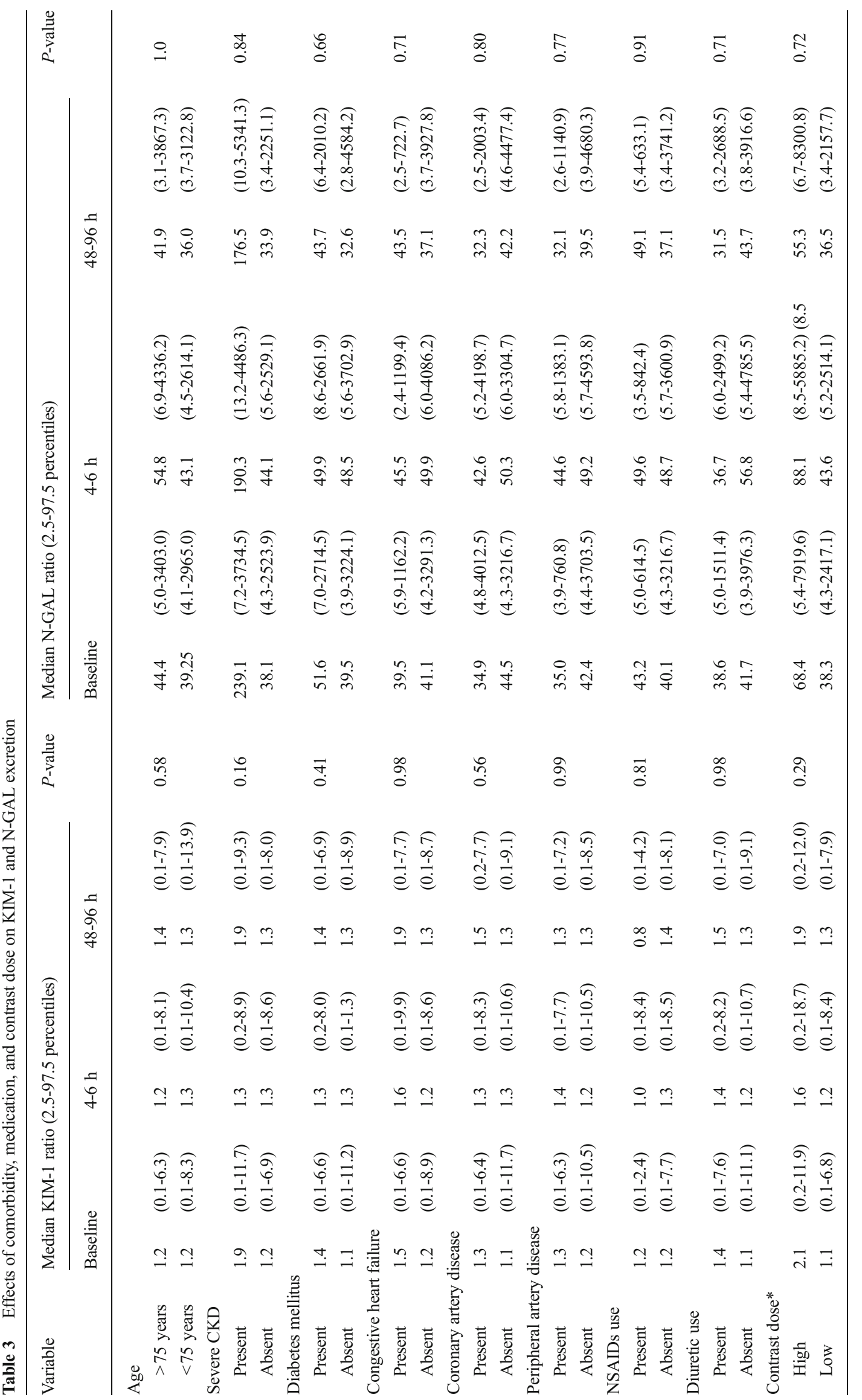


Table 4 Test characteristics of KIM-1 and N-GAL excretion for the diagnosis of contrast-induced acute kidney injury after $48-96 \mathrm{~h}$

\begin{tabular}{lll}
\hline Biomarker & AUC & $95 \%$ CI \\
\hline KIM-1 ratio & & \\
$\quad$ Baseline & 0.47 & $(0.34-0.60)$ \\
4-6 h post-CE-CT & 0.52 & $(0.36-0.66)$ \\
48-96 h post-CE-CT & 0.50 & $(0.34-0.65)$ \\
N-GAL ratio & & \\
Baseline & 0.55 & $(0.36-0.65)$ \\
4-6 h post-CE-CT & 0.53 & $(0.35-0.70)$ \\
48-96 h post-CE-CT & 0.63 & $(0.45-0.80)$ \\
\hline
\end{tabular}

$A U C$ area under the curve; $C E-C T$ intravenous contrast media-enhanced $\mathrm{CT}$

analyzing both KIM-1 and N-GAL expression post-CE-CT. We analyzed these two established biomarkers of CI-AKI within adequate time frames, as indicated by previous studies [11, 14]. Moreover, due to the prospective study design, all relevant information on co-morbidity influencing our outcomes was available for analysis. However, some aspects of our study require comment. We included a population with mainly moderate CKD since only $7 \%$ of patients had a baseline eGFR value of less than $30 \mathrm{ml} / \mathrm{min}$. Therefore, we can't exclude the possibility of some extent of tubular injury post CE-CT in patients with severe CKD. However, that group reflects only $10 \%$ of CKD patients undergoing CE-CT in daily practice [36]. Moreover, our sample size was too small to study differences in KIM-1 and N-GAL expression between different types of contrast media.

In conclusion, KIM-1 and N-GAL excretion were unaffected by CE-CT in both patients with and without CI-AKI. This study suggests that CE-CT can probably be safely performed in patients with $\mathrm{CKD}$, and challenges the creatinine based definition of CI-AKI.

Acknowledgments The scientific guarantor of this publication is Professor A.J. Rabelink. The authors of this manuscript declare no relationships with any companies, whose products or services may be related to the subject matter of the article. The authors state that this work has not received any funding. One of the authors has significant statistical expertise. Institutional review board approval was obtained. Written informed consent was obtained from all subjects (patients) in this study. Methodology: prospective, randomised controlled trial, multicenter study.

Open Access This article is distributed under the terms of the Creative Commons Attribution Noncommercial License which permits any noncommercial use, distribution, and reproduction in any medium, provided the original author(s) and the source are credited.

\section{References}

1. Seeliger E, Sendeski M, Rihal CS, Persson PB (2012) Contrastinduced kidney injury: mechanisms, risk factors, and prevention. Eur Heart J 33:2007-2015
2. Stacul F, van der Molen AJ, Reimer P, Webb JA, Thomsen HS, Morcos SK et al (2011) Contrast induced nephropathy: updated ESUR Contrast Media Safety Committee guidelines. Eur Radiol 21:2527-2541

3. Kooiman J, Pasha SM, Zondag W, Sijpkens YW, van der Molen AJ, Huisman MV et al (2012) Meta-analysis: serum creatinine changes following contrast enhanced CT imaging. Eur J Radiol 81:2554-2561

4. Moos SI, van Vemde DN, Stoker J, Bipat S (2013) Contrast induced nephropathy in patients undergoing intravenous (IV) contrast enhanced computed tomography (CECT) and the relationship with risk factors: A meta-analysis. Eur J Radiol 2013

5. Slocum NK, Grossman PM, Moscucci M, Smith DE, Aronow HD, Dixon SR et al (2012) The changing definition of contrast-induced nephropathy and its clinical implications: insights from the Blue Cross Blue Shield of Michigan Cardiovascular Consortium (BMC2). Am Heart J 163:829-834

6. Russo D, Minutolo R, Cianciaruso B, Memoli B, Conte G, De NL (1995) Early effects of contrast media on renal hemodynamics and tubular function in chronic renal failure. J Am Soc Nephrol 6:1451-1458

7. Waikar SS, Bonventre JV (2009) Creatinine kinetics and the definition of acute kidney injury. J Am Soc Nephrol 20:672-679

8. Katzberg RW, Newhouse JH (2010) Intravenous contrast mediuminduced nephrotoxicity: is the medical risk really as great as we have come to believe? Radiology 256:21-28

9. McDonald RJ, McDonald JS, Bida JP, Carter RE, Fleming CJ, Misra $S$ et al (2013) Intravenous contrast material-induced nephropathy: causal or coincident phenomenon? Radiology 267:106-118

10. Alharazy SM, Kong N, Saidin R, Abdul Gafor AH, Maskon O, Mohd M, et al (2013) Neutrophil Gelatinase-Associated Lipocalin as an Early Marker of Contrast-Induced Nephropathy After Coronary Angiography. Angiology

11. Bachorzewska-Gajewska H, Malyszko J, Sitniewska E, Malyszko JS, Pawlak K, Mysliwiec M et al (2007) Could neutrophil-gelatinaseassociated lipocalin and cystatin $\mathrm{C}$ predict the development of contrast-induced nephropathy after percutaneous coronary interventions in patients with stable angina and normal serum creatinine values? Kidney Blood Press Res 30:408-415

12. Lenhard DC, Pietsch H, Sieber MA, Ernst R, Lengsfeld P, Ellinghaus $P$ et al (2012) The osmolality of nonionic, iodinated contrast agents as an important factor for renal safety. Invest Radiol 47:503-510

13. Ling W, Zhaohui N, Ben H, Leyi G, Jianping L, Huili D et al (2008) Urinary IL-18 and NGAL as early predictive biomarkers in contrastinduced nephropathy after coronary angiography. Nephron Clin Pract 108:c176-c181

14. Malyszko J, Bachorzewska-Gajewska H, Poniatowski B, Malyszko JS, Dobrzycki S (2009) Urinary and serum biomarkers after cardiac catheterization in diabetic patients with stable angina and without severe chronic kidney disease. Ren Fail 31:910-919

15. McCullough PA, Williams FJ, Stivers DN, Cannon L, Dixon S, Alexander P et al (2012) Neutrophil gelatinase-associated lipocalin: a novel marker of contrast nephropathy risk. Am J Nephrol 35:509-514

16. Shaker OG, El-Shehaby A, El-Khatib M (2010) Early diagnostic markers for contrast nephropathy in patients undergoing coronary angiography. Angiology 61:731-736

17. Kooiman J, Sijpkens YW, de Vries JP, Brulez HF, Hamming JF, van der Molen AJ, et al (2014) A randomized comparison of 1-h sodium bicarbonate hydration versus standard peri-procedural saline hydration in patients with chronic kidney disease undergoing intravenous contrast-enhanced computerized tomography. Nephrol Dial Transplant

18. Ralib AM, Pickering JW, Shaw GM, Devarajan P, Edelstein CL, Bonventre JV et al (2012) Test characteristics of urinary biomarkers depend on quantitation method in acute kidney injury. J Am Soc Nephrol 23:322-333

19. American College of Radiology (2012). Manual on Contrast Media v8 
20. McDonald JS, McDonald RJ, Comin J, Williamson EE, Katzberg RW, Murad MH et al (2013) Frequency of acute kidney injury following intravenous contrast medium administration: a systematic review and meta-analysis. Radiology 267:119-128

21. Bansal GJ, Darby M (2009) Measurement of change in estimated glomerular filtration rate in patients with renal insufficiency after contrast-enhanced computed tomography: a case-control study. J Comput Assist Tomogr 33:455-459

22. Bruce RJ, Djamali A, Shinki K, Michel SJ, Fine JP, Pozniak MA (2009) Background fluctuation of kidney function versus contrast-induced nephrotoxicity. AJR Am J Roentgenol 192: 711-718

23. McDonald JS, McDonald RJ, Carter RE, Katzberg RW, Kallmes DF, Williamson EE (2014) Risk of Intravenous Contrast Materialmediated Acute Kidney Injury: A Propensity Score-matched Study Stratified by Baseline-estimated Glomerular Filtration Rate. Radiology 130775

24. Newhouse JH, Kho D, Rao QA, Starren J (2008) Frequency of serum creatinine changes in the absence of iodinated contrast material: implications for studies of contrast nephrotoxicity. AJR Am J Roentgenol 191:376-382

25. Lacquaniti A, Buemi F, Lupica R, Giardina C, Mure G, Arena A et al (2013) Can neutrophil gelatinase-associated lipocalin help depict early contrast material-induced nephropathy? Radiology 267:86-93

26. Filiopoulos V, Biblaki D, Lazarou D, Chrisis D, Fatourou M, Lafoyianni S et al (2013) Plasma neutrophil gelatinase-associated lipocalin (NGAL) as an early predictive marker of contrast-induced nephropathy in hospitalized patients undergoing computed tomography. Clin Kidney J 28:8

27. Kooiman J, Pasha SM, Zondag W, Sijpkens YW, van der Molen AJ, Huisman MV, et al (2011) Meta-analysis: Serum creatinine changes following contrast enhanced CT imaging. Eur J Radiol
28. Waanders F, van Timmeren MM, Stegeman CA, Bakker SJ, van Goor H (2010) Kidney injury molecule-1 in renal disease. J Pathol 220:7-16

29. Ichimura T, Asseldonk EJ, Humphreys BD, Gunaratnam L, Duffield JS, Bonventre JV (2008) Kidney injury molecule-1 is a phosphatidylserine receptor that confers a phagocytic phenotype on epithelial cells. J Clin Invest 118:1657-1668

30. Mishra J, Ma Q, Prada A, Mitsnefes M, Zahedi K, Yang J et al (2003) Identification of neutrophil gelatinase-associated lipocalin as a novel early urinary biomarker for ischemic renal injury. J Am Soc Nephrol 14:2534-2543

31. Smertka M, Chudek J (2012) Using NGAL as an early diagnostic test of acute kidney injury. Ren Fail 34:130-133

32. Fukumoto Y, Tsutsui H, Tsuchihashi M, Masumoto A, Takeshita A (2003) The incidence and risk factors of cholesterol embolization syndrome, a complication of cardiac catheterization: a prospective study. J Am Coll Cardiol 42:211-216

33. Saklayen MG, Gupta S, Suryaprasad A, Azmeh W (1997) Incidence of atheroembolic renal failure after coronary angiography. A prospective study. Angiology 48:609-613

34. Vuurmans T, Byrne J, Fretz E, Janssen C, Hilton JD, Klinke WP et al (2010) Chronic kidney injury in patients after cardiac catheterisation or percutaneous coronary intervention: a comparison of radial and femoral approaches (from the British Columbia Cardiac and Renal Registries). Heart 96:1538-1542

35. Kooiman J, Le Haen PA, Gezgin G, de Vries JP, Boersma D, Brulez HF et al (2013) Contrast-induced acute kidney injury and clinical outcomes after intra-arterial and intravenous contrast administration: risk comparison adjusted for patient characteristics by design. Am Heart J 165:793-799

36. Kim SM, Cha RH, Lee JP, Kim DK, Oh KH, Joo KW et al (2010) Incidence and outcomes of contrast-induced nephropathy after computed tomography in patients with CKD: a quality improvement report. Am J Kidney Dis 55:1018-1025 